\title{
CHARACTERIZING THE COOL KOIs. IV. KEPLER-32 AS A PROTOTYPE FOR THE FORMATION OF COMPACT PLANETARY SYSTEMS THROUGHOUT THE GALAXY
}

\author{
Jonathan J. Swift ${ }^{1}$, John Asher Johnson ${ }^{1,2,3,6}$, Timothy D. Morton ${ }^{1,3}$, Justin R. Crepp ${ }^{4}$, Benjamin T. Montet ${ }^{1}$, \\ Daniel C. FABRYCKY ${ }^{5,7}$, AND Philip S. Muirhead ${ }^{1}$ \\ ${ }^{1}$ Department of Astrophysics, California Institute of Technology, MC 249-17, Pasadena, CA 91125, USA; jswift@astro.caltech.edu \\ ${ }^{2}$ Division of Geological and Planetary Sciences, California Institute of Technology, Pasadena, CA 91125, USA \\ ${ }^{3}$ NASA Exoplanet Science Institute (NExScI), CIT Mail Code 100-22, 770 South Wilson Avenue, Pasadena, CA 91125, USA \\ ${ }^{4}$ Department of Physics, 225 Nieuwland Science Hall, University of Notre Dame, Notre Dame, IN 46556, USA \\ ${ }^{5}$ Department of Astronomy and Astrophysics, University of California, Santa Cruz, Santa Cruz, CA 95064, USA \\ Received 2012 October 15; accepted 2012 December 11; published 2013 January 29
}

\begin{abstract}
The Kepler space telescope has opened new vistas in exoplanet discovery space by revealing populations of Earth-sized planets that provide a new context for understanding planet formation. Approximately $70 \%$ of all stars in the Galaxy belong to the diminutive M dwarf class, several thousand of which lie within Kepler's field of view, and a large number of these targets show planet transit signals. The Kepler M dwarf sample has a characteristic mass of $0.5 M_{\odot}$ representing a stellar population twice as common as Sun-like stars. Kepler-32 is a typical star in this sample that presents us with a rare opportunity: five planets transit this star, giving us an expansive view of its architecture. All five planets of this compact system orbit their host star within a distance one-third the size of Mercury's orbit, with the innermost planet positioned a mere 4.3 stellar radii from the stellar photosphere. New observations limit possible false positive scenarios, allowing us to validate the entire Kepler-32 system making it the richest known system of transiting planets around an $\mathrm{M}$ dwarf. Based on considerations of the stellar dust sublimation radius, a minimum mass protoplanetary nebula, and the near period commensurability of three adjacent planets, we propose that the Kepler-32 planets formed at larger orbital radii and migrated inward to their present locations. The volatile content inferred for the Kepler-32 planets and order of magnitude estimates for the disk migration rates suggest that these planets may have formed beyond the snow line and migrated in the presence of a gaseous disk. If true, then this would place an upper limit on their formation time of $\sim 10$ Myr. The Kepler-32 planets are representative of the full ensemble of planet candidates orbiting the Kepler $\mathrm{M}$ dwarfs for which we calculate an occurrence rate of $1.0 \pm 0.1$ planet per star. The formation of the Kepler-32 planets therefore offers a plausible blueprint for the formation of one of the largest known populations of planets in our Galaxy.
\end{abstract}

Key words: methods: statistical - planetary systems - planets and satellites: detection - planets and satellites: formation - stars: individual (KID 9787239/KOI-952/Kepler-32)

Online-only material: color figures

\section{BACKGROUND}

Before the discovery of exoplanets around main-sequence stars two decades ago, models of planet formation were based on a solitary example: our own solar system. Despite the discovery of hundreds, if not thousands of additional planets in the years since then, observational and theoretical efforts have focused on the formation of planets around Sun-like stars. However, the Sun is not a typical star. Seventy percent of stars in the Galaxy are dwarfs of the M spectral class ("M dwarfs" or "red dwarfs"; Bochanski et al. 2010), with masses that are only $\sim 10 \%-50 \%$ the mass of the Sun, much cooler temperatures, and different evolutionary histories. These differences likely result in different formation and evolutionary histories for their planets. For example, both Doppler and transit surveys have revealed a paucity of gas giant planets around $\mathrm{M}$ dwarfs, and a relative overabundance of planets with masses less than that of Neptune (Howard et al. 2012). This is in contrast to the high gas giant occurrence rate around stars more massive than the Sun (Johnson et al. 2010a). These correlations between stellar mass and gas giant occurrence are likely a consequence of the lower disk masses around $\mathrm{M}$ dwarfs, which result in less raw material

\footnotetext{
${ }^{6}$ Sloan Fellow.

7 Hubble Fellow.
}

available for planet building (Laughlin et al. 2004; Kennedy \& Kenyon 2008).

Roughly 5500 of the 160,000 stars targeted by NASA's Kepler mission are $\mathrm{M}$ dwarfs with a mass distribution skewed toward the high-mass end of the spectral class (see Section 5.1 for details). Of these stars, 66 show at least one periodic planetary transit signal. Aside from a single outlier - the hot Jupiter system around KOI-254 (Johnson et al. 2012) - the ensemble of 100 planet candidates around these $\mathrm{M}$ dwarfs have radii ranging from $\sim 0.5$ to $3 R_{\oplus}$ and semimajor axes within about a few tenths of an astronomical unit (Muirhead et al. 2012a). These compact planetary configurations around the lowest mass stars (e.g., Muirhead et al. 2012b) offer a number of advantages: the signal-to-noise ratio $(\mathrm{S} / \mathrm{N})$ of the transit signals of closein planets is boosted due to the increased number of transits per observing period (Gould et al. 2003); transit depths are larger for a given planet radius allowing detections of ever smaller planets (Nutzman \& Charbonneau 2008); and the reduced temperatures and luminosities of their host stars lead to equilibrium temperatures comparable to Earth's, despite their extreme proximity to their stars (Kasting et al. 1993; Tarter et al. 2007).

The Kepler M dwarf planets have been calculated to have an occurrence rate a factor of $\sim 3$ higher than for solartype stars with occurrence rates increasing as planet size and 
stellar mass decrease (Mann et al. 2012; Howard et al. 2012). Microlensing studies also suggest a high planet occurrence around low-mass stars (Cassan et al. 2012). However, the large errors on these results and the fact that microlensing surveys probe planets at larger separations from their host stars than transit surveys complicate the comparison between samples. The high frequency of small planets around low-mass stars is compounded by the fact that lower mass stars are more common than solar-type stars. Therefore, the mechanisms by which small planets form around the lowest mass stars determine the characteristics of the majority of planets currently known to exist in our Galaxy.

Kepler-32 is an M1V star with half the mass and radius of the Sun, roughly two-thirds the Sun's temperature, and 5\% of its luminosity (Muirhead et al. 2012a). While showing five distinct transit signals, to date only two of the planets have been validated, Kepler-32 b and c, from the timing variations of their transit signals (TTVs; Fabrycky et al. 2012a). The Kepler-32 host star and its planets are typical of the full Kepler M dwarf sample, except for the chance alignment of this system with respect to our line of sight that offers a unique and expansive view of its dynamical architecture. To capitalize on this chance alignment, we bring to bear a suite of ground-based observations using the W. M. Keck Observatory and the Robo-AO system on the Palomar 60 inch telescope (Baranec et al. 2012) to validate and characterize the system in detail. We follow this with an in-depth analysis that allows us to place tight constraints on the formation and evolution of the Kepler-32 planets, which in turn has implications for the formation of planets around early $\mathrm{M}$ dwarfs in general.

Since all planet parameters are derived directly from the physical characteristics of the host star, we start by refining the stellar properties in Section 2. High spatial resolution images and optical spectra are then used by our false positive statistical analyses to validate the remaining three transit signals from the Kepler data in Section 3. These above analyses provide the foundation for an accurate characterization of the Kepler-32 planets followed by an investigation into the formation and evolutionary history of this system implied by its observed architecture in Section 4. We then turn to a discussion of the ensemble of Kepler M dwarf planets in Section 5 and argue that the formation pathway deduced for Kepler-32 offers a plausible blueprint for the formation of planets around the smallest stars, and thus the majority of all stars throughout the Galaxy.

\section{KEPLER-32 STELLAR PROPERTIES}

The observed stellar properties for Kepler-32 are summarized in Table 1. It was originally listed in the Kepler Input Catalog (KIC) with an effective temperature of $3911 \mathrm{~K}$, below the $4500 \mathrm{~K}$ threshold where the photometric method for determining stellar parameters is deemed reliable (Brown et al. 2011). Follow-up, medium-resolution infrared spectroscopy presented by Muirhead et al. (2012a) revised and refined the KIC values to $T_{\text {eff }}=3727_{-58}^{+102}$ and $[\mathrm{Fe} / \mathrm{H}]=0.04 \pm 0.13$.

In this work, we supplement these measurements with an independent estimation of the stellar mass, radius, and metallicity using the broadband photometric method presented by Johnson et al. (2012). This method evaluates the posterior probability distribution of each stellar parameter conditioned on the observed apparent magnitudes and colors of the star listed in the KIC using model relationships such as the Delfosse et al. (2000) mass-luminosity relation and the West et al. (2005) color-spectral-type relation. Our Markov Chain Monte Carlo
Table 1

Observed Stellar Properties

\begin{tabular}{lcr}
\hline \hline Parameter & Value & Ref. \\
\hline$\alpha(\mathrm{J} 2000)$ & $19: 51: 22.18$ & 1 \\
$\delta(\mathrm{J} 2000)$ & $46^{\circ} 34^{\prime} 27^{\prime \prime}$ & 1 \\
$\mu_{\alpha}\left(\mathrm{mas} \mathrm{yr}^{-1}\right)$ & -8 & 1 \\
$\mu_{\delta}\left(\mathrm{mas} \mathrm{yr}^{-1}\right)$ & 20 & 1 \\
$K_{P}$ & 15.801 & 1 \\
$A_{V}$ & 0.154 & 1 \\
$g$ & 17.251 & 1 \\
$r$ & 15.913 & 1 \\
$V$ & $16.452^{\mathrm{a}}$ & 2 \\
$J$ & $13.616 \pm 0.023$ & 3 \\
$H$ & $12.901 \pm 0.024$ & 3 \\
$K_{s}\left(\mathrm{~km} \mathrm{~s}^{-1}\right)$ & $12.757 \pm 0.024$ & 3 \\
$v_{\text {rad }}\left(\mathrm{m}^{-1}\right.$ & $-32.5 \pm 0.5$ & 4 \\
\hline
\end{tabular}

Notes.

${ }^{\text {a }}$ Converted from $g$ and $r$.

References. (1) Borucki et al. 2011b; (2) Jester et al. 2005; (3) Brown et al. 2011 ; (4) this work.

analysis yields $M_{\star}=0.57 \pm 0.06 M_{\odot}, R_{\star}=0.53 \pm 0.04 R_{\odot}$, and $[\mathrm{Fe} / \mathrm{H}]=-0.05 \pm 0.17$.

We combine the results of the above analyses with those of Borucki et al. (2011a), Muirhead et al. (2012a), and Fabrycky et al. (2012a) by weighted mean to obtain the final values used in this work presented in Table 2 . We adopt $10 \%$ errors on the Borucki et al. (2011a) values for stellar mass and radius. For the effective temperature, we construct probability distribution functions and compute the mean and $1 \sigma$ errors numerically to account for the asymmetric errors in the Muirhead et al. (2012a) value. Using the Delfosse et al. (2000) $K$-band mass-luminosity relationship, we find that our final stellar mass and its uncertainty give a distance modulus of $7.407 \pm 0.098$, corresponding to a distance of $d=303 \pm 14$ pc.

\subsection{Stellar Age}

It is difficult to measure the age of field $\mathrm{M}$ dwarfs unassociated with known moving groups or clusters because their global stellar properties change little over the course of their mainsequence lifetime. However, Kepler-32 has a slow rotation rate of $P=37.8 \pm 1.2$ days measured from the modulation of the light curve by star spots (Fabrycky et al. 2012a), implying that it is old. To estimate the age of Kepler-32, we use the age equation from Barnes (2010),

$$
t=\frac{\tau}{k_{C}} \ln \left(\frac{P}{P_{0}}\right)+\frac{k_{I}}{2 \tau}\left(P^{2}-P_{0}^{2}\right) .
$$

The dimensionless constants $k_{C}=0.646$ days $\mathrm{Myr}^{-1}$ and $k_{I}=452 \mathrm{Myr}$ day $^{-1}$ are determined observationally, the convection turnover time is estimated as $\tau=k_{I} / 2 \cdot f^{2}(B-V)$, where we use $f(B-V)=0.77(B-V-0.4)^{0.6}$ (Barnes 2007) and $(B-V)=1.5$ (converted from $g-r$ using Jester et al. 2005) to give $\tau=155$ days. Using the median value of the initial spin period, $P_{0}=2.81$, as required to produce the observed rotation rates for $\approx 0.6 M_{\odot}$ stars in the Praesepe cluster (Agüeros et al. 2011), we derive an age of 2.7 Gyr for Kepler-32. The large scatter in observed rotation rates for Praesepe members used here to calibrate $P_{0}$ suggests that the age of Kepler-32 could be as young as $2.3 \mathrm{Gyr}$ or as old as $3.7 \mathrm{Gyr}$. For this work, we will assume that the age of Kepler-32 is greater than 2 Gyr. 
Table 2

Derived Stellar Properties

\begin{tabular}{lccccc}
\hline \hline Parameter & KIC $^{\mathrm{a}}$ & ${\mathrm{M} 12^{\mathrm{b}}}^{\mathrm{b}}$ & F12 & This Work & Adopted Values \\
\hline$T_{\text {eff }}(\mathrm{K})$ & $3911 \pm 200$ & $3727_{-58}^{+102}$ & $3900 \pm 200$ & $\ldots$ & $3793_{-74}^{+80}$ \\
$M_{\star}\left(M_{\odot}\right)$ & $0.49 \pm 0.05$ & $0.52 \pm 0.04$ & $0.58 \pm 0.05$ & $0.57 \pm 0.06$ & $0.54 \pm 0.02$ \\
$R_{\star}\left(M_{\odot}\right)$ & $0.56 \pm 0.06$ & $0.50 \pm 0.04$ & $0.53 \pm 0.04$ & $0.53 \pm 0.04$ & $0.53 \pm 0.02$ \\
{$[\mathrm{Fe} / \mathrm{H}]$} & $-0.056 \pm 0.2$ & $0.04 \pm 0.13$ & $0 \pm 0.4$ & $-0.05 \pm 0.17$ & $-0.01 \pm 0.09$ \\
$d(\mathrm{pc})$ & $\ldots$ & $\ldots$ & $\ldots$ & $303 \pm 14$ & $303 \pm 14$ \\
age $(\mathrm{Gyr})$ & $\ldots$ & $\ldots$ & $\ldots$ & $\sim 2.7$ & $>2$ \\
\hline
\end{tabular}

Notes.

a Brown et al. (2011).

b Muirhead et al. (2012a).

c Fabrycky et al. (2012a).

\section{VALIDATION OF TRANSIT SIGNALS}

Two of the Kepler-32 planets (b and c) have been validated previously using signatures of their mutual dynamical interactions (Fabrycky et al. 2012a). The other three transit signals have hitherto retained the status of planet candidates. The probabilities for Kepler planet candidates to be astrophysical false positives, such as blended stellar eclipsing binaries, are generally low (Morton \& Johnson 2011), and may even be negligible in multiply transiting systems (Lissauer et al. 2012). Diffraction limited images in the Kepler band made with the Robo-AO system on the Palomar 60 inch telescope (Baranec et al. 2012) show no evidence of blended companions for a $5 \sigma$ contrast of $\Delta z \approx 3.5$ at 0.5 and $\Delta z \approx 4.5$ at $1^{\prime \prime}$. High-resolution optical spectroscopy with the HIgh-Resolution Echelle Spectrometer (HIRES) on Keck I and high-resolution adaptive optics (AO) imaging in the near infrared using the NIRC2 camera on Keck II place stringent constrains on the probabilities of various false positive scenarios, allowing a probabilistic validation of the transit signals using the procedure of Morton (2012).

\subsection{Keck I/HIRES Spectroscopy}

We obtained spectra of Kepler-32 at the Keck Observatory using the HIRES spectrograph (Vogt et al. 1994) on Keck I with the standard observing setup used by the California Planet Survey (Johnson et al. 2010b), which covers a wavelength range from $3640 \AA$ to $7820 \AA$. Because of the star's faint visual magnitude, we used the $\mathrm{C} 2$ decker corresponding to a projected size of $14 . \prime 0 \times 00^{\prime \prime} 851$ to allow sky subtraction and a resolving power of $R=55,000$. We obtained two observations of Kepler32 (UT 2011 June 18 and UT 2012 March 13), both with an exposure time of $700 \mathrm{~s}$, each resulting in an S/N of 20 at $6500 \AA$.

To measure the systemic radial velocity of Kepler-32 and to check for evidence of a second set of stellar lines in the spectrum, we performed a cross-correlation analysis with respect to a HIRES spectrum of a similar star (HIP 86961: $V_{\text {rad }}=-28.9 \pm 0.4 \mathrm{~km} \mathrm{~s}^{-1}$; Nidever et al. 2002) using the methodology described by Johnson et al. (2010a). Our analysis yields a systemic radial velocity of $-32.5 \pm 0.5 \mathrm{~km} \mathrm{~s}^{-1}$. No second set of lines are evident above the noise floor in the crosscorrelation function, allowing us to rule out stellar companions with $V>10 \mathrm{~km} \mathrm{~s}^{-1}$ and $V$-band brightnesses within $2 \mathrm{mag}$.

\subsection{Keck II/NIRC2 Adaptive Optics Imaging}

We performed AO imaging using the NIRC2 instrument on the Keck II telescope on UT 2011 June 24 to rule out false positive scenarios involving blended sources. With a Kepler

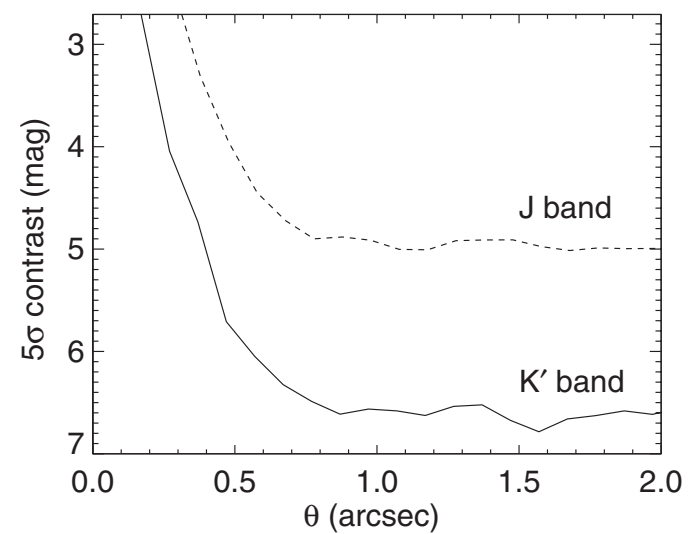

Figure 1. $J$ - and $K^{\prime}$-band contrast curves derived from our Keck II/NIRC2 adaptive optics imaging constrain false positive scenarios involving blended sources.

bandpass magnitude $K_{P}=15.80$, Kepler-32 is relatively faint for natural guide star observations. Nevertheless, we were able to close the AO system control loops on the star with a frame rate of $41 \mathrm{~Hz}$. With sufficient counts in each wavefront sensor subaperture, a stable lock was maintained for the duration of the observations.

We acquired two sets of dithered images using the NIRC2 medium camera (plate scale $=20$ mas pix $^{-1}$ ) a sequence of nine images in the $J$ filter (central wavelength $=1.25 \mu \mathrm{m}$ ), and a sequence of nine images in the $K^{\prime}$ filter (central wavelength $=$ $2.12 \mu \mathrm{m}$ ). Each image had an exposure time of $20 \mathrm{~s}$, resulting in a total on-source integration time of $180 \mathrm{~s}$ per filter. To process the data, hot pixels were removed, the sky background was subtracted, and the images were aligned then co-added. We measured the contrast achieved by NIRC 2 AO imaging by comparing the peak intensity of the star in the final processed image to the intensity of residual scattered starlight at small angular separations. Specifically, we calculate the standard deviation of flux, $\sigma$, within a box of size $3 \times 3$ FWHM, where the FWHM is the point-spread function full width at half-maximum spatial scale (also the size of a speckle). The standard deviation is evaluated at numerous locations close to the star and the results are azimuthally averaged to estimate the local radial contrast profile. Figure 1 shows the $5 \sigma$ contrast curves based on each reduced AO image. The full instrument field of view is $20^{\prime \prime} \times 20^{\prime \prime}$, which corresponds to 5 Kepler pixels on a side. No obvious contaminants were identified within $\Delta K^{\prime}=6.5$ at a separation of 0.'7 or farther from Kepler-32. 


\subsection{Kepler Light Curves}

Our light curve data come from the Kepler space telescope, which is conducting a continuous photometric monitoring campaign of a target field near the constellations Cygnus and Lyra. A $0.95 \mathrm{~m}$ aperture Schmidt telescope feeds a mosaic CCD photometer with a $10^{\circ} \times 10^{\circ}$ field of view (Koch et al. 2010; Borucki et al. 2011b). Data reduction and analysis is described by Jenkins et al. (2010a, 2010b) and photometric and astrometric data were made publicly available as part of the 2012 July 28 public data release. We downloaded the data from the Multimission Archive at STScI (MAST), and we use the pipeline-corrected light curves from Quarters 0-9 (Batalha et al. 2012).

These light curves were aggressively detrended to take out all astrophysical variation by first masking out all transit signals and then sequentially fitting a low-order polynomial to the time series data in two day chunks. These detrended data were then folded for each Kepler-32 d, e, and f, with the other planet signals masked according to the ephemeris of Fabrycky et al. (2012a). These folded transit signals were used for the false positive probability (FPP) analysis. The binned photometric data are shown in Figure 2 with the trapezoidal fits used to assess the transit shape overlaid.

\subsection{False Positive Probability Analysis}

To calculate the relative probabilities of astrophysical false positive and bona fide transiting planet scenarios, we follow the methodology of Morton (2012), which compares the observed transit shapes to those of simulated false positive populations, subject to the available observational constraints. Although occurrence rates may be as high as $10 \%$ for planets in the radius bins corresponding to KOI-952.03,.04, and .05 (Howard et al. 2012), we assume a very conservative occurrence rate of $1 \%$.

Figure 3 summarizes these FPP results for all three signals. Despite our conservative estimates of planet occurrence rate, and without accounting for the fact that Kepler-32 b and $c$ have already been confirmed to be coplanar transiting planets, which would further decrease the FPPs by a factor of about 10, we estimate the FFPs for KOI-952.03, .04, and .05 to all be less than $0.3 \%$. We therefore consider all five photometric signals to be fully validated planet transits, making Kepler-32 the richest known system of transiting planets around an $\mathrm{M}$ dwarf, and we assign the names Kepler-32 d, e, and $\mathrm{f}$ to the former candidates KOI-952.03, .04, and .05, respectively. Verification of the transit signals allows us to take full advantage of the fortuitous alignment of the Kepler-32 system with respect to our line of sight, and in the next section we look deeper into the details and implications of its specific configuration.

\section{THE KEPLER-32 PLANETARY SYSTEM}

The increased accuracy with which we derive the stellar parameters for Kepler-32 (see Table 2) leads to more precise planetary parameters, which we outline in Table 3 . The planets of Kepler-32 have radii that are similar to values found in the solar system, from below Earth size up to about $70 \%$ of Neptune. However, the planetary system of Kepler-32 has a remarkably distinct dynamical architecture in comparison. Figure 4 shows the relative sizes of the planets and their orbits, along with labels denoting their periods, semimajor axes, and period commensurabilities rounded to the nearest integer. The five planets of this system orbit within 0.13 AU from the star, or approximately one-third of Mercury's semimajor axis. The
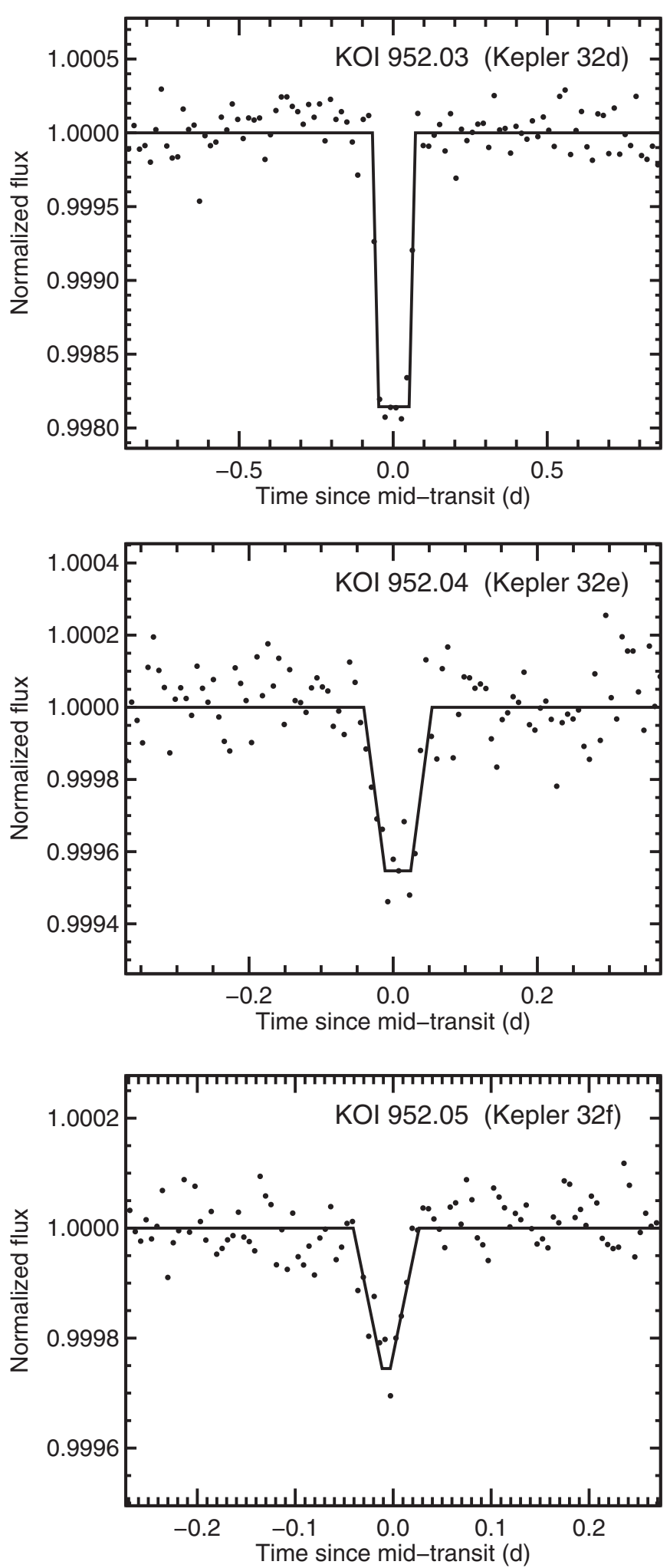

Figure 2. Detrended, folded, and binned Kepler light curves for the three planets that we validate with our FPP analysis. The trapezoidal fits from the analysis of Morton (2012) are overlaid.

outermost planet lies within a region where the stellar insolation is similar to Earth's, while at the other extreme, the innermost planet orbits only 4.3 stellar radii from the photosphere of Kepler-32. The three middle planets - termed e, b, and c in order of increasing semimajor axis-exhibit period ratios within $2 \%$ of a $1: 2: 3$ commensurability. 
KOI 952.03
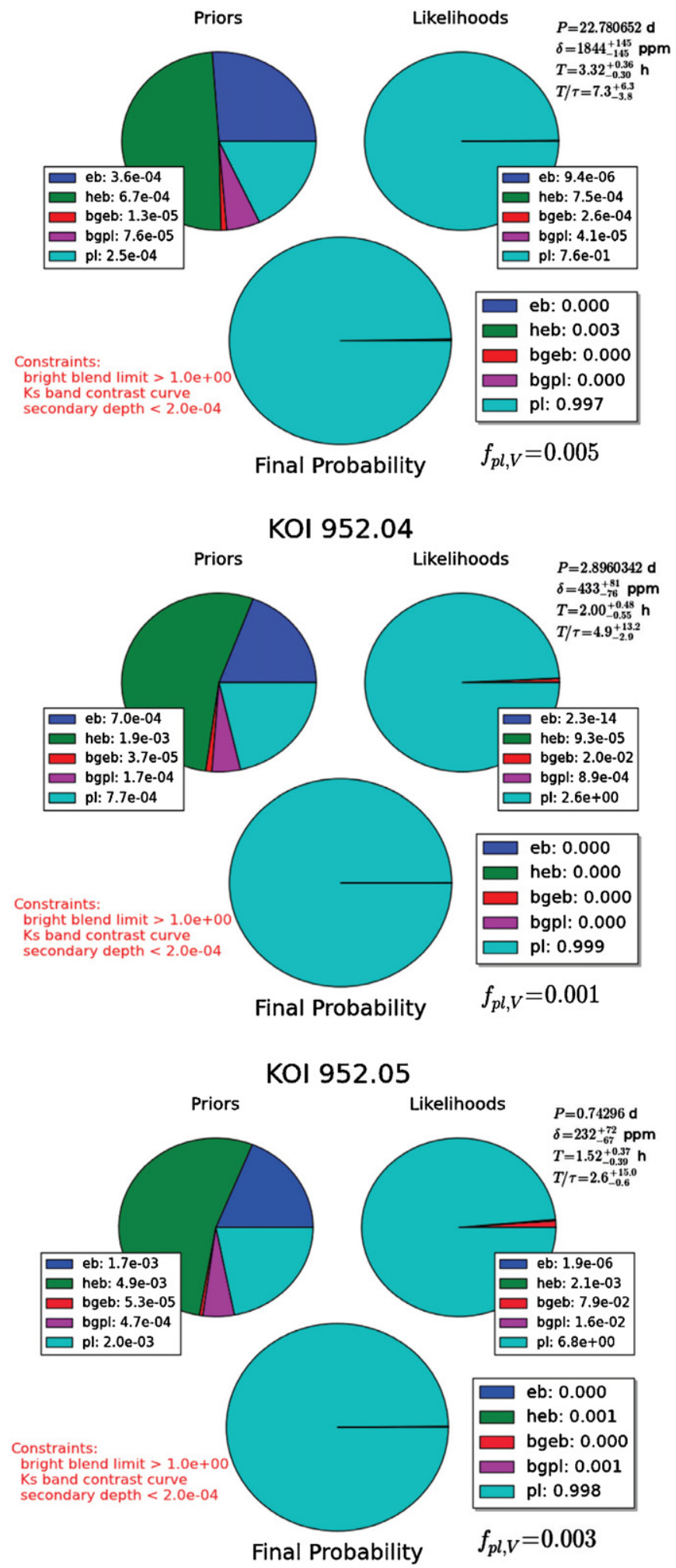

Figure 3. Relative strength of each possible scenario in our false positive calculation-eclipsing binary (eb), hierarchical binary (heb), background eclipsing binary (bgeb), background planet (bpl), and planet (pl) - is shown pictorially for our priors (upper left chart), likelihood (upper right chart), and final probability (lower chart) for each KOI-952.03 (top panel), .04 (middle panel), and .05 (bottom panel). Observational constraints used in the calculation are stated in red (lower left) and the trapezoidal fit parameters are shown (upper right) in each panel. The quantity $f_{\mathrm{pl}, V}$ is the smallest value of the planet occurrence that would result in validation for the given transit signal.

(A color version of this figure is available in the online journal.)
Table 3

Derived Planet Properties

\begin{tabular}{lccccr}
\hline \hline KOI & Kepler & $\begin{array}{c}P^{\mathrm{a}} \\
(\text { days })\end{array}$ & $\begin{array}{c}a \\
(\mathrm{AU})\end{array}$ & $\begin{array}{c}R_{p} \\
\left(R_{\oplus}\right)\end{array}$ & $\begin{array}{c}T_{\mathrm{eq}}{ }^{\mathrm{b}} \\
(\mathrm{K})\end{array}$ \\
\hline 952.05 & $32 \mathrm{f}$ & $0.74296(7)$ & $0.0130(2)$ & $0.81(5)$ & 1100 \\
952.04 & $32 \mathrm{e}$ & $2.8960(3)$ & $0.0323(5)$ & $1.5(1)$ & 680 \\
952.01 & $32 \mathrm{~b}$ & $5.9012(1)$ & $0.0519(8)$ & $2.2(1)$ & 530 \\
952.02 & 32c & $8.7522(3)$ & $0.067(1)$ & $2.0(2)$ & 470 \\
952.03 & $32 \mathrm{~d}$ & $22.7802(5)$ & $0.128(2)$ & $2.7(1)$ & 340 \\
\hline
\end{tabular}

Notes.

${ }^{\text {a }}$ Fabrycky et al. (2012a).

b Assuming a Bond albedo $\alpha=0.3$.

\subsection{Tidal Evolution}

The proximity of the Kepler-32 planets to their host star imply that their dynamics have been significantly altered due to tidal forces induced by the host star over the age of the system. Using an initial spin period of $10 \mathrm{hr}$, a tidal dissipation factor of 100 , a rigidity factor of $3 \times 10^{11}$, and a density profile similar to Earth such that the moment of inertia $I \approx 0.5 M_{p} R_{p}^{2}$, we derive the tidal locking timescales for the Kepler-32 planets to all be $\lesssim 1$ Myr (Murray \& Dermott 1999). This timescale depends linearly on the dissipation factor, $Q$, which is highly uncertain for exoplanets. However, for a reasonable range of $Q$ values ranging up to $10^{5}$, it can be expected that the rotation periods of all of the planets in the Kepler-32 system are equal to their orbital period barring spin-orbit resonances.

Tidal forces from the host star will also damp the eccentricities of the planets in direct proportion to $Q$ (Murray \& Dermott 1999). For $Q<10^{4}$, the inner planets will have no free (primordial) eccentricity. However, even for a low $Q$, the tidal damping time for Kepler-32 d, the most distant planet, is approximately $4 \mathrm{Gyr}$, comparable to the age of the system. The free eccentricities of Kepler- $32 \mathrm{~b}$ and $\mathrm{c}$ have recently been estimated to have non-zero values from the phase of the TTV signals (Lithwick et al. 2012; Wu \& Lithwick 2012). This suggests high $Q$ values for these planets, consistent with their moderate inferred densities (see Section 4.2).

Contrary to the trend for Kepler pairs to lie longward of mean motion resonance (Fabrycky et al. 2012b), Kepler-32 b and c lie $1.1 \%$ shortward of resonance. The high $Q$ values suggested above would limit dissipative mechanisms that may spread their orbits (Lithwick \& Wu 2012; Batygin \& Morbidelli 2013). Also, Kepler-32 b is near a 2:1 resonance with Kepler32 e. However, our $n$-body simulation of Section 4.4 suggests planet e has too small an effect on Kepler-32 b to account for this unusual configuration.

\subsection{Mass and Density Estimates}

As mentioned above, Kepler-32 b and c were validated based on the TTVs observed due to their mutual interactions (Fabrycky et al. 2012a). The amplitude of the TTV signals specify upper limits to their masses of 6.6 and $8.4 M_{\oplus}$, implying densities of less than 3.4 and $5.7 \mathrm{~g} \mathrm{~cm}^{-3}$ for $\mathrm{b}$ and $\mathrm{c}$, respectively (Lithwick et al. 2012). However, the TTV phases are measured to be $\sim 45^{\circ}$ from 0 and $\pi$, implying a small free eccentricity. A correction for this effect by Wu \& Lithwick (2012) reduces the estimated masses for Kepler-32 b and $\mathrm{c}$ by factors of 0.51 and 0.45 . With these corrections, the masses and densities for Kepler-32 $\mathrm{b}$ and $\mathrm{c}$ are 3.4 and $3.8 M_{\oplus}$ and 1.7 and $2.6 \mathrm{~g} \mathrm{~cm}^{-3}$. The nominal masses for Kepler-32 b and c imply mass-radius relationships 


\section{Kepler 32 Planetary System}

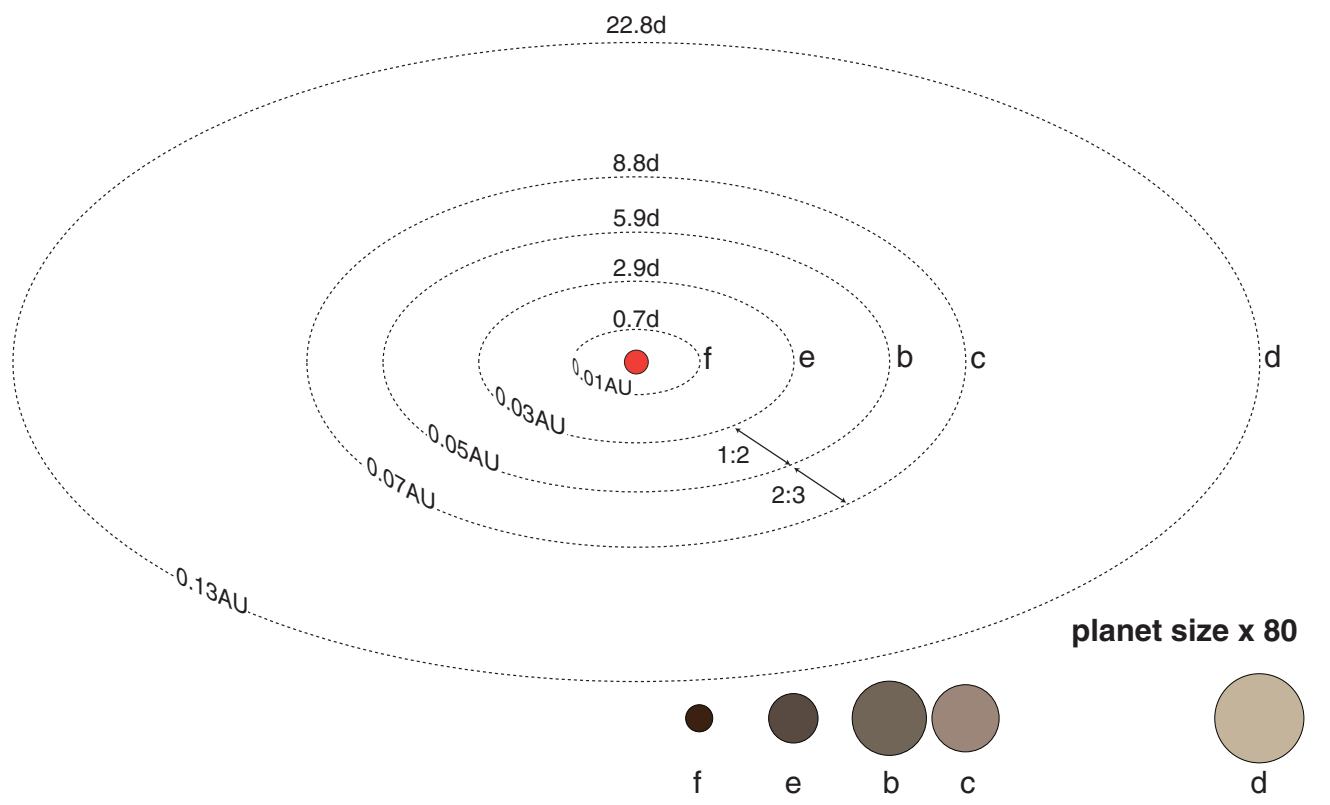

Figure 4. Depiction of the Kepler-32 planetary system with the star and orbits drawn to scale. The relative sizes of the planets are shown at the bottom of the figure scaled up by a factor of 80 in relation to their orbits.

(A color version of this figure is available in the online journal.)

of $M \propto R^{\gamma_{p}}$, with $\gamma_{p}=1.5-1.9$, similar to the value of 2.06 for the six solar system planets bounded by Mars and Saturn (Lissauer et al. 2011).

The above stated densities imply that Kepler-32 b and c are composed of a significant amount of volatiles. Using Equations (7) and (8) of Fortney et al. (2007), we find that if Kepler-32 b and c had no atmospheres, then they would be expected to contain $\sim 96 \%$ and $\sim 56 \%$ volatiles. However, given the equilibrium temperatures of Kepler-32 b and c, a large fraction of their volatile content likely exists in the form of an atmosphere.

\subsection{Atmospheric Evolution}

The proximity of the Kepler-32 planets to their host star suggest significant atmospheric evolution due to evaporation, outgassing, or both processes. The equilibrium temperature of Kepler-32 $\mathrm{f}$ is $\sim 1100 \mathrm{~K}$ and its radius is measured to be $0.81 R_{\oplus}$. For a planet this small with such a high equilibrium temperature, the atmospheric mass fraction would have to be very small, $\sim 10^{-5}$ (Rogers et al. 2011). Using an extreme ultraviolet luminosity of Kepler- $32, L_{\mathrm{EUV}} \approx 10^{26.6}$ (Hodgkin \& Pye 1994), and following Lecavelier Des Etangs (2007) using a conservative mass-loss efficiency of $\epsilon_{\mathrm{UV}}=0.1$, we derive an atmospheric mass loss of $\sim 10^{8} \mathrm{~g} \mathrm{~s}^{-1}$. Thus, the timescale to lose its atmosphere is more than 100 times shorter than the age of the Kepler-32 system. We therefore conclude that the Kepler-32 f contains no atmosphere.

Given the size and equilibrium temperature of Kepler-32 e, its atmospheric mass fraction must also be small, $M_{a} / M_{p} \sim 10^{-4}$, while the present-day atmospheric mass-loss rate is between $10^{7}$ and $10^{8} \mathrm{~g} \mathrm{~s}^{-1}$. The timescale for the complete loss of the Kepler32 e atmosphere is calculated to be between 0.2 and 2 Gyr. Therefore, Kepler-32 e must have lost a significant fraction of any atmosphere it started with.

The total atmospheric mass loss for the other three planets is at least $\sim 10^{-4} M_{\oplus}$ for reasonable choices of planetary mass.
If these planets have relatively low density cores (ice and rock) and started out with large atmospheres, then they could have suffered considerable atmospheric evolution due to the heating by Kepler-32. Thus, the observed sizes of the Kepler-32 planets are likely determined in part by the extreme ultraviolet and $\mathrm{X}$-ray luminosity of their host star. However, the mass estimates from Section 4.2 suggest that Kepler-32 b is $10 \%$ less massive than Kepler-32 c while being 10\% larger and 25\% closer to Kepler-32, hinting that the mass-radius relation for the Kepler32 planets is not determined solely by a simple atmospheric evolution model.

\subsection{Kepler-32 Planetary System Architecture}

The physical characteristics of the Kepler-32 planets are summarized in Table 3 and the remarkably compact and orderly architecture of the system is shown schematically in Figure 4. As mentioned above, three of the planets lie within $2 \%$ of a $1: 2: 3$ period commensurability. Kepler- 32 e and $b$ have a period ratio of 2.038 , which is $1.9 \%$ longward of commensurability, while Kepler-32 b and c have a period ratio of 1.483 , or $1.1 \%$ shortward of commensurability.

Planets within a mean motion resonance can stray a few percent from commensurability and maintain the libration of resonant angles (Murray \& Dermott 1999). However, without detailed knowledge of the individual orbits, it is not possible to determine with certainty if a planet pair is in a resonant configuration. Therefore, we assess the significance of the near commensurability of Kepler-32 e, b, and c using a probabilistic argument.

We randomly populate five planet systems with periods between the inner and outermost planets in the Kepler-32 system, enforcing separations larger than $2 \sqrt{3}$ for every pair of neighboring planets (Gladman 1993) and larger than 9 for chains of planets (Chambers et al. 1996; Smith et al. 2009; Lissauer et al. 2011) in units of mutual Hill radii. In this section, a mass-radius relationship of $M \propto R^{2.06}$ (Lissauer et al. 2011) is 
adopted for consistency. The final ensemble of systems provide a baseline against which we can gauge the significance of the Kepler-32 architecture. The occurrence of period ratios in our randomly drawn sample that lie within the observed period ratio of either Kepler-32 e and b, or b and c, is fairly high, $11 \%$ and $12 \%$, respectively. However, the fraction of systems arranged in a chain involving any combination of $1: 2$ or $2: 3$ near-commensurabilities is $1.9 \% \pm 0.5 \%$. This suggests that the architecture we observe today reflects the result of dynamical evolution rather than a result of chance.

Given the compactness of the Kepler-32 system, we expand on the dynamical stability simulations of Fabrycky et al. (2012a) by considering all five planets. Kepler- $32 \mathrm{f}$ is very likely on a circular orbit and lies $>30$ mutual Hill radii away from the nearest planet. It therefore is not expected to have any bearing on the stability of the system (Smith \& Lissauer 2009) and is not included in the following analysis.

Using the Mercury6 software (Chambers 1999) employing the hybrid symplectic Bulirsch-Stoer integrator, we integrate the Kepler-32 system for $30 \mathrm{Myr}$, or $\sim 500$ million orbits of the outermost planet. Our initial conditions were set according to the ephemeris of Fabrycky et al. (2012a) with an initial time step of one-tenth the orbital period of the innermost planet (in this case, Kepler-32 e). We began with zero eccentricity and random inclinations drawn uniformly between the minimum and maximum values that would produce a transit from our vantage point. We did not include any tidal effects. The eccentricities and inclinations varied stably with small amplitudes. Figure 5 shows the inclination evolution resampled at $100 \mathrm{kyr}$ time steps and histograms of the eccentricities measured every $100 \mathrm{yrs}$ over the 30 Myr simulation for the outer four planets.

\subsection{The Formation and Evolution of the Kepler-32 Planetary System}

The long-term stability evident in our simulation of Section 4.4 allows us to view the present-day architecture of the Kepler-32 planets as representing the state of the system at the end of its formation epoch, 10-100 Myr after the formation of the star. We now investigate what can be learned about the formation of the Kepler-32 planets by looking backward from their end state.

\subsubsection{The Kepler-32 Protoplanetary Nebula}

Planets form from flattened disks of dust and gas circulating around protostars, and the masses and sizes of these protoplanetary disks as well as their lifetimes and accretion rates are constrained from observations (see Williams \& Cieza 2011). The surface density profiles are typically assumed to follow a power-law form $\Sigma_{d} \propto a^{-\gamma}{ }^{8}$. The value of $\gamma$ is often taken to be $3 / 2$ based on estimates of the minimum mass solar nebula (MMSN; Weidenschilling 1977; Hayashi 1981). The MMSN is constructed by smoothing the planets of the solar system over their respective domains and correcting for solar abundance to recreate a minimal surface density profile from which the solar system could have formed. Modern observations constrain the surface density profile in the outer disk ( $\gtrsim 40 \mathrm{AU})$ to be on average shallower than this, $\gamma \approx 0.4-1.0$ (Andrews \& Williams 2007; Isella et al. 2009; Andrews et al. 2009), while the form

\footnotetext{
8 Here and throughout, we use $a$ to denote both the orbital radius of circumstellar material and the semimajor axis of the planets. The Kepler planets typically have low eccentricities (Wu \& Lithwick 2012), minimizing ambiguity.
}

Kepler 32b
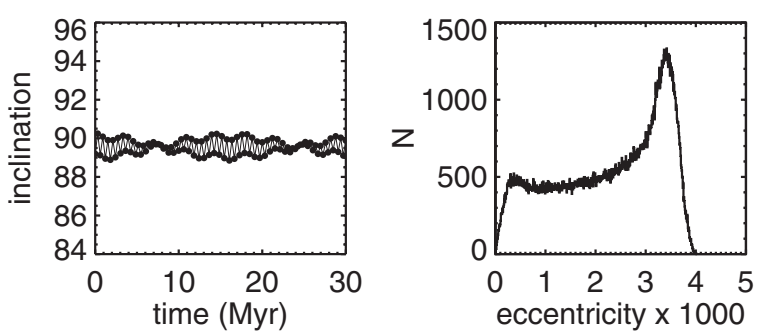

Kepler 32c
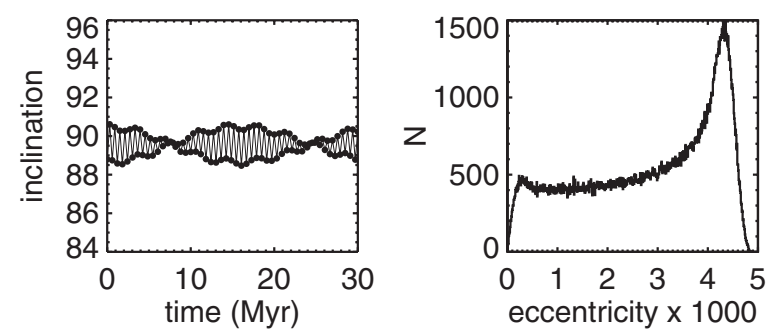

Kepler 32d
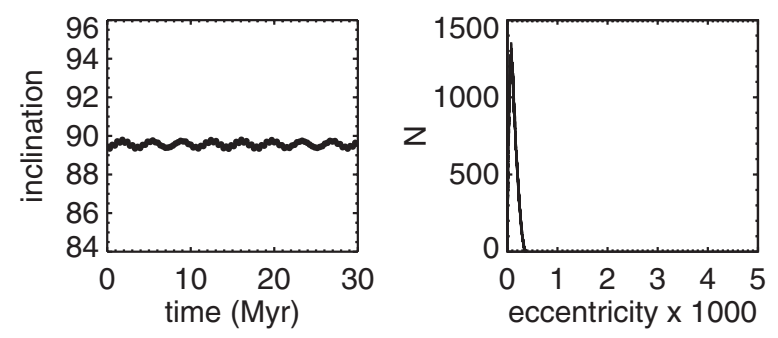

Kepler $32 \mathrm{e}$
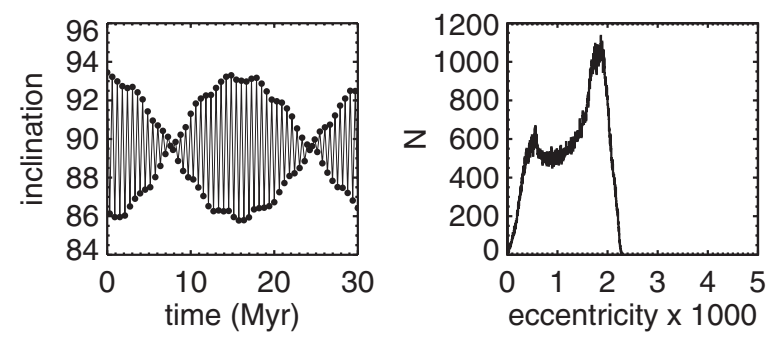

Figure 5. Time evolution of the inclinations sampled every $100 \mathrm{kyr}$ and the distributions of eccentricities for the Kepler-32 planets sampled every $100 \mathrm{yrs}$ from our $n$-body simulation. Kepler- $32 \mathrm{f}$ was not included in the simulation due to its dynamical isolation from the rest of the planetary system (see the text).

of the surface density profile in the inner disk remains largely unconstrained.

We can construct a "minimum mass protoplanetary nebula" for Kepler-32 in a similar fashion. Since we do not have mass estimates for all of the planets in the Kepler-32 system, we use the mass-radius relationship for solar system planets $M \propto R^{2.06}$ (Lissauer et al. 2011), consistent with the nominal masses from Section 4.2 to within $50 \%$. Assuming a gas-to-dust ratio of 100 and a rocky core mass fraction of $50 \%$ implies that the protoplanetary nebula of Kepler-32 contained $\approx 3 M_{\text {Jup }}$ of gas between 0.013 and $0.13 \mathrm{AU}$ of the host star.

This translates to a very high surface density, $\approx 5 \times 10^{5} \mathrm{~g} \mathrm{~cm}^{-2}$, in the inner regions of the disk that is incongruous with the masses and surface density profiles in the outer regions of disks as measured with (sub)-millimeter interferometry (Andrews \& Williams 2007; Isella et al. 2009; Andrews et al. 2009). Indeed, if this surface density is used to scale the median 


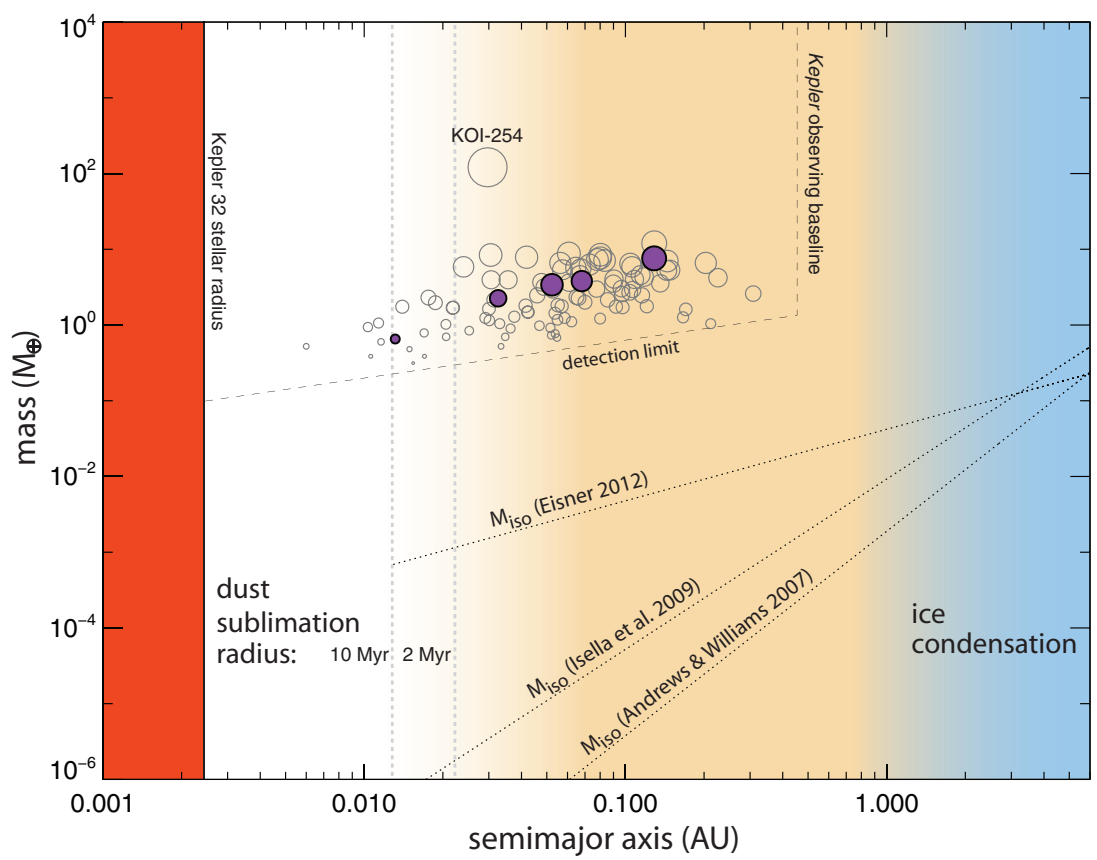

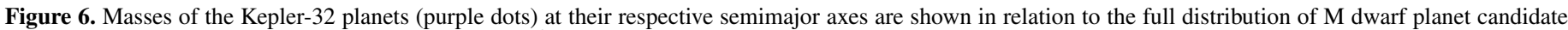

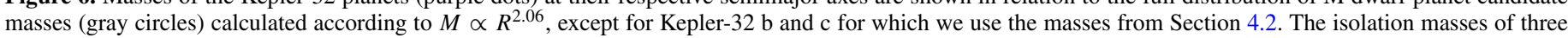

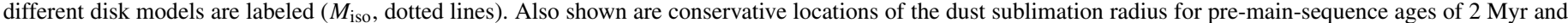

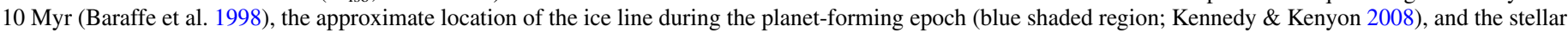

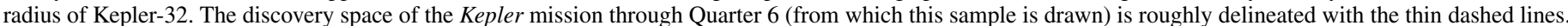

best-fit values of the disk radius and surface density profile index for stars less than $1 M_{\odot}$ in the Andrews et al. (2009) sample $-R_{d}=126 \mathrm{AU}$ and $\gamma=0.9-$ then the total mass of the Kepler-32 protoplanetary nebula would be orders of magnitude greater than the star! This implies one of three possibilities: the disk surface density deviated significantly from a single power law in the inner regions with a large pile-up of material near the disk inner radius (see, e.g., Chiang \& Laughlin 2012); the material that formed the planets came from elsewhere in the disk; or the planets themselves formed elsewhere and were transported to their present-day locations.

\subsubsection{Oligarchic Growth and the Isolation Mass}

The gravitational influence of the host star poses another barrier to super-Earth-sized planets forming within a tenth of an AU. The amount of material accessible to a growing planetary embryo during the oligarchic phase of growth is estimated by the "isolation mass" (Lissauer 1987). This quantity is derived by calculating the amount of mass available to an oligarch within a disk of planetesimals. We choose the radius of gravitational influence to be four Hill radii based on the approximate spacing of isolated oligarchs in numerical simulations (Kokubo \& Ida 1998) to obtain

$$
M_{\text {iso }}=\frac{\left[16 \pi a^{2} \Sigma(a)\right]^{3 / 2}}{\left(3 M_{\star}\right)^{1 / 2}},
$$

where $a$ is the radial distance from the star, $M_{\star}$ is the mass of the star, and $\Sigma(a)$ is disk surface density profile. To calculate values for $M_{\text {iso }}(a)$, we again use the median surface density profile of the M-type stars in the Andrews et al. (2009) sample, the median values of the M-type stars in the Isella et al. (2009) sample with $\gamma=0.5$ and $R_{d}=260 \mathrm{AU}$, and also the Eisner (2012) model with a median disk radius of $R_{d}=250 \mathrm{AU}$ and a surface density profile with fixed $\gamma=1.37$ based on theoretical arguments.
Figure 6 shows that the isolation mass computed for these surface density models are $\gtrsim 3$ orders of magnitude too small to account for the mass in the Kepler-32 planets. While the planetary embryos can undergo an additional phase of growth through subsequent mergers that increase their mass by an order of magnitude (e.g., Chambers \& Wetherill 1998), it would be difficult to achieve an additional factor of $\sim 100$ growth during this phase (see also Lissauer 2007). The total amount of growth in this late phase growth is limited to the total amount of mass present in the disk at the outset, which is addressed in the previous section.

The migration of planetesimals from outside this region also offers a possibility for late time growth of the Kepler-32 planets (Hansen \& Murray 2012). However, the gravitational stirring of the planetesimals can cause radial displacements of the planetary embryos away from period commensurability. The magnitude of this effect has recently been used to place limits on the amount of mass contained in orbit-crossing planetesimals at the end stages of planet formation for closely spaced, low-inclination multi-planet Kepler systems (Moore et al. 2012). These limits of $\sim 1 M_{\oplus}$ are too low to contribute significantly to the discrepancy between the isolation mass and the final mass of the Kepler-32 planets.

This corroborates the above conclusions that the Kepler-32 protoplanetary disk either contained a disproportionately large amount of mass within $0.1 \mathrm{AU}$, or that the formation of the Kepler-32 planets followed a more complex formation and evolution history.

\subsubsection{The Dust Sublimation Radius}

Planets form from dust in protoplanetary disks. However, at small orbital radii, the stellar irradiation raises the dust temperature beyond the sublimation point, clearing these regions of the raw materials for planet formation. Low-mass stars such as 


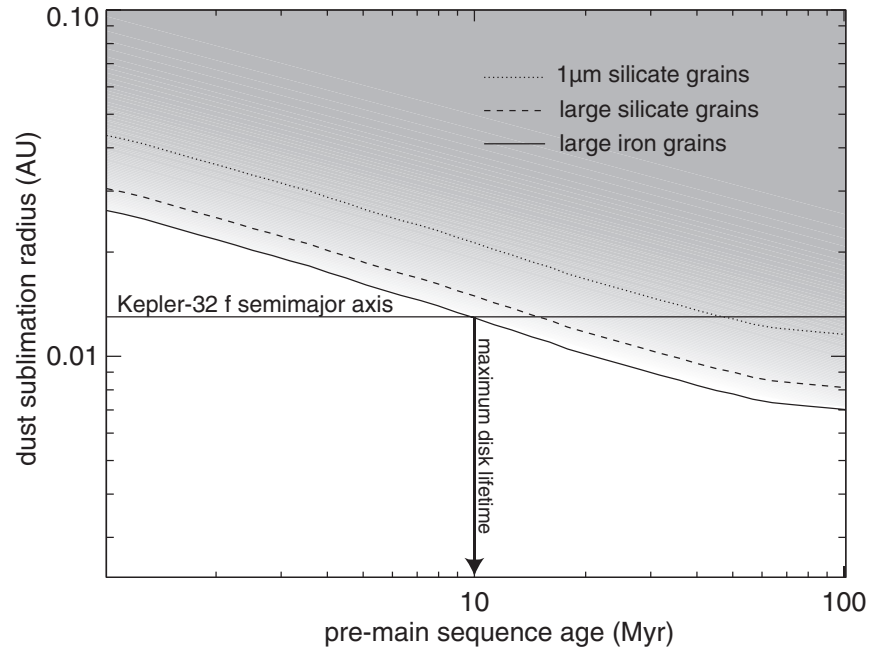

Figure 7. Dust sublimation radius of Kepler-32 as a function of pre-mainsequence age according to the models of Baraffe et al. (1998). The solid curve is for large iron grains ( $T_{\text {sub }}=1908 \mathrm{~K}$ and $\left.\epsilon=1\right)$ the dashed curve is for large silicate (olivine) grains $\left(T_{\mathrm{sub}}=1774 \mathrm{~K}\right.$ and $\left.\epsilon=1\right)$, and the dotted curve is for $1 \mu \mathrm{m}$ silicate (olivine) grains within a lower density environment $\left(T_{\text {sub }}=1570 \mathrm{~K}\right.$ and $\left.\epsilon=0.58\right)$. All curves cross the present-day semimajor axis of Kepler-32 $\mathrm{f}$ after the typical lifetime of a protoplanetary disk.

Kepler-32 begin pre-main-sequence evolution with luminosities much larger than their main-sequence values and take $>10 \mathrm{Myr}$ to contract to the point when hydrogen burning begins. The sublimation radius at these early epochs is thus further from the star than one would conclude from the present-day luminosity of the star.

The dust sublimation radius is given as

$$
R_{\text {sub }}=0.034\left(\frac{1500}{T_{\text {sub }}}\right)^{2}\left(\frac{L_{\star}}{L_{\odot}}\left(2+\frac{1}{\epsilon}\right)\right)^{1 / 2}
$$

(Isella et al. 2009) where $T_{\text {sub }}$ is the sublimation temperature of the dust, $L_{\star}$ is the stellar luminosity, and $\epsilon$ is a measure of the cooling efficiency of the grains, with larger grains having higher efficiencies leading to smaller sublimation radii (Isella et al. 2006). Figure 7 shows the evolution of $R_{\text {sub }}$ as a function of the pre-main-sequence age of a $0.5 M_{\odot}$ star based on the stellar evolution models of Baraffe et al. (1998).

Even if it is conservatively assumed that the dust is in large grains $(\epsilon=1)$ and composed of pure iron with a sublimation temperature $T_{\text {sub }}=1908 \mathrm{~K}$ (Pollack et al. 1994), the sublimation radius does not move inward of Kepler-32 f's orbit until after 9.8 Myr of pre-main-sequence evolution. For smaller grains made of silicates and for less dense environments, the dust sublimation radius lies beyond the semimajor axis of Kepler$32 \mathrm{f}$ until after $\sim 50 \mathrm{Myr}$, and beyond the present-day orbit of Kepler-32 e for $~ 3$ Myr of pre-main-sequence evolution. This poses a serious challenge to in situ formation, particularly since the dusty disks from which planets form only last $\lesssim 10 \mathrm{Myr}$ before they are drained, disrupted, or evaporated away (Williams \& Cieza 2011).

\subsubsection{In Situ Formation versus Formation then Migration}

The above investigation into the structure of the Kepler-32 planetary system as well as the history and evolution of the host star present meaningful constraints on its formation. To form Kepler-32 $\mathrm{f}$ and e where we view them today requires that they formed from planetesimals scattered inward from the wider orbit planets. This kind of formation scenario would be different than the theory described by Hansen \& Murray (2012), where scattered planetesimals accrete onto pre-existing oligarchs from an early phase of planet formation. Rather, Kepler-32 $\mathrm{f}$ and $\mathrm{e}$ would have had to have formed from scattered planetesimals in a region largely devoid of solid material. It is not clear how feasible this would be, and requires an investigation beyond the scope of this work. An additional challenge for in situ formation of the Kepler-32 planets is to explain the near period commensurabilities of Kepler-32 e, b, and c, which we have shown to be statistically significant beyond pure chance.

On the other hand, a scenario in which the Kepler-32 planets formed further out in the disk and then migrated to their present locations offers a natural explanation for (1) the large amount of planetary mass within $0.1 \mathrm{AU}$ of this $0.54 M_{\odot}$ star, (2) the position of Kepler-32 $\mathrm{f}$ within the dust sublimation radius of the star for $\sim 10 \mathrm{Myr}$ of pre-main-sequence evolution, and (3) the near period commensurabilities between three of the planets. The high volatile content inferred for Kepler-32 b and c is also indirect evidence that these planets may have formed beyond the snow line at $\sim 1$ AU (Kennedy \& Kenyon 2008). The difficulties in applying this concept lie in understanding the mechanisms of migration through a disk of gas and planetesimals. While a full, rigorous analysis of these possibilities is also beyond the scope of this article, we look to some order of magnitude calculations of different migration mechanisms for insight.

\subsubsection{Migration}

The compact and coplanar architecture of the Kepler-32 system favors migration through a disk rather than through planet-planet interactions. Interactions with either a gaseous disk or a disk of planetesimals can cause radial migration of planetary orbits (see Kley \& Nelson 2012, for a review). Planets massive enough to open a gap in the protoplanetary disk are coupled to the viscous evolution of the disk, while less massive planets migrate more quickly due to larger disk torques.

Using a generalized criterion for gap formation in a viscous disk (Crida et al. 2006, Equation (15)), we find that none of the Kepler-32 planets would have opened a gap in their protoplanetary disk assuming a constant disk flaring term $a / H=0.05$, where $H$ is the scale height, a viscosity parameter $\alpha=10^{-3}$, and using the nominal masses for Kepler-32 b and c (Section 4.2) and $M_{p} \propto R_{p}^{2.06}$ otherwise. We therefore restrict our discussion of migration through a gaseous disk to the "type I" mechanism (Goldreich \& Tremaine 1979; Lin \& Papaloizou 1979).

Assuming a protoplanetary disk with a power-law surface density profile, $\Sigma=\Sigma_{0} a^{-\gamma}$, and a midplane temperature profile, $T \propto a^{-\beta_{T}}$, we estimate the migration rate through a gaseous disk following Kley \& Nelson (2012)

$$
\frac{d a}{d t}=\Gamma_{\text {tot }} \frac{2}{m_{p}}\left(\frac{a_{p}}{G M_{\star}}\right)^{1 / 2},
$$

where the $p$ subscripts refer to planet quantities, and $\Gamma_{\text {tot }}$ is the total torque on the planet from Linblad, corotation, and horseshoe torques

$$
\Gamma_{\text {tot }}=\left[-1.36+0.62 \gamma+0.43 \beta_{T}+1.36(3 / 2-\gamma)\right] \Gamma_{0}
$$

(also see Masset et al. 2006), and

$$
\Gamma_{0}=\left(\frac{m_{p}}{M_{\star}}\right)^{2}\left(\frac{H}{a_{p}}\right)^{-2} \Sigma_{p} a_{p}^{4} \Omega_{p}^{2} .
$$


As a baseline model, we use a disk surface density profile with $\gamma=1$, which extends from the present-day location of Kepler-32 f out to $200 \mathrm{AU}$, contains a total of $10 \%$ of the stellar mass, and has a midplane temperature profile with $\beta_{T}=3 / 4$. This disk model yields type I migration rates $d a / d t \sim-0.07,-0.3,-0.7,-0.9$, and $-1 \mathrm{AU}\left(10^{4} \mathrm{yr}\right)^{-1}$ for Kepler-32 $\mathrm{f}$ through Kepler-32 c, respectively, with the migration rate scaling as $d a / d t \propto m_{p} \Sigma_{0} a_{p}^{3 / 2-\gamma}$. For these calculated rates, the timescale for these planets to have migrated from beyond the snow line at $\sim 1 \mathrm{AU}$ (Kennedy \& Kenyon 2008) to their present-day locations is short, $\tau_{\mathrm{mig}} \sim 10^{4} \mathrm{yr}$, compared to typical disk lifetimes.

The increasing mass of the Kepler-32 planets as a function of semimajor axis naturally produces convergent migration for type I torques in a smooth disk, a necessary but not sufficient condition for resonant capture. To estimate the probability for resonant capture, we perform an order of magnitude calculation comparing the resonance crossing times to the libration time for the resonances. The resonance widths and libration times are estimated using the pendulum model for an interior resonance (Murray \& Dermott 1999, Sections 8.6 and 8.7). For the $2: 3$ resonance of Kepler-32 $\mathrm{b}$ and $\mathrm{c}$, the libration period is $T_{\text {lib }} \sim 10 \mathrm{yr}$ and the width is estimated to be $\Delta a_{\text {res }} \sim 0.009 \mathrm{AU}$ using the mean eccentricity of Kepler-32 $b$ from the simulations of Section 4.4. With a convergence rate of $0.2 \mathrm{AU}\left(10^{4} \mathrm{yr}\right)^{-1}$, we find that the resonance crossing time is roughly 40 libration periods. Following the same procedure for the 1:2 resonance of Kepler-32 e and b, we find that the resonance crossing is of the order of 10 libration periods.

Shallower surface density profiles produce slower convergence rates, increasing the probability of resonant capture, while steeper profiles produce faster convergence, shortening resonance crossing times and decreasing the probability for capture. For $\gamma=1 / 2$, we find that the resonance crossing times are on the order of $10^{3}$ libration periods for both resonances, and for $\gamma=3 / 2$ these times are shortened to $\lesssim 1$. The resonance crossing times in units of libration periods is a weakly increasing function of semimajor axis for our simple model, going as $a_{p}^{1 / 4}$.

Therefore, we find that the Kepler-32 planets could have been captured into resonance while migrating through a gaseous disk if the surface density profile had $\gamma \lesssim 1$, consistent with modern observations of protoplanetary disks (Andrews et al. 2009). The probability for capture is a weak function of semimajor axis, and it is thus also feasible for the Kepler-32 planets to have been caught in resonance further out in the disk and to have moved inward in lock step (e.g., Cresswell \& Nelson 2008). How the planets may have stopped their migration before falling onto the star remains an outstanding question in this scenario.

It is also possible that the Kepler-32 planets migrated through a planetesimal disk (Levison et al. 2007) rather than the gas dominated disk required for type I migration. We calculate the order of magnitude rates for planetesimal migration using the equations of Bromley \& Kenyon (2011) assuming a gas to dust ratio of 100 and that all solids are in the form of planetesimals. We find migration rates of $d a / d t \sim$ $-0.44,-1.1,-1.7,-2.1,-3.2 \mathrm{AU}\left(10^{8} \mathrm{yr}\right)^{-1}$, roughly four orders of magnitude slower than type I. This slow migration rate would favor resonance capture and may circumvent the need for an efficient stopping mechanism. However, it may be difficult to migrate these planets in lock step from much further out in the disk, as the inner planets could stir the planetesimal disk reducing the migration efficiency at that location for the next further out planet (Kirsh et al. 2009; Bromley \& Kenyon
2011). Also, if Kepler-32 b and c were migrating at such slow rates, then it is likely that they would have ended up in a 1:2 resonance rather than the more compact $2: 3$ resonance. These details may limit the distances over which planetesimal migration could have acted to produce the observed architecture of Kepler-32.

From these analyses, migration through a gaseous disk produces a more favorable situation for transporting the Kepler32 planets from far enough out in the disk to reconcile the difficulties with in situ formation outlined above. However, further investigation into the migration of multi-planet systems through both gas and planetesimal disks is needed to draw more definitive conclusions.

\section{DISCUSSION}

The analyses of the preceding sections provide evidence that the Kepler-32 planets formed further out in their protoplanetary disk from where we see them today and migrated convergently into their present locations. The high inferred volatile content of Kepler-32 b and c, and the order of magnitude calculations for the migration rates of the Kepler-32 planets suggest that these planets have formed beyond the snow line in the presence of gas. If true, then the Kepler-32 planets would have necessarily formed within $\sim 10 \mathrm{Myr}$, the timescale over which gas survives in protoplanetary disks (Williams \& Cieza 2011). This formation history stands in contrast to the formation of the terrestrial planets in the solar system, which are commonly thought to have formed in situ in a gas-free environment on a $100 \mathrm{Myr}$ timescale (Wetherill 1990). The possibility of constraining the timescale for the formation of the Kepler-32 planets motivates further investigation into the migration of multi-planet systems.

Our conclusions about the formation of the Kepler-32 planets rely on a detailed characterization of a single system not possible for the full ensemble of Kepler's M dwarf planets. However, both the Kepler-32 star and its planets are representative of this full ensemble, providing important clues regarding the formation of the full sample of Kepler M dwarf planet candidates.

\subsection{The Ensemble of Kepler M Dwarf Planets}

Our sample of M dwarfs was drawn from the catalog of Kepler Objects of Interest (KOIs) given by Batalha et al. (2012) using a color-magnitude cut of $K_{P}>14$ and $K_{p}-J>2$ (Mann et al. 2012). This results in 5499 stars from the KIC catalog observed with Kepler, however, there are only 4682 with finite, non-zero photometric precision values for at least one quarter between Q0 and Q6. Since the Kepler sample is magnitude limited, the sample is skewed toward the most massive M dwarfs, peaking around $0.5 M_{\odot}$ (see Figure 10). Two giant planet candidates are present in this sample. One is a bona fide giant planet confirmed with radial velocity data (KOI-254; Johnson et al. 2012). The other, KOI-1902, we found to be a false positive based on its transit profile. We scrutinized the light curves of several of the other apparent outliers by hand. We reject two KOIs in the list, KOI-531 and KOI-1152, on account of deep secondary transits, and two others, KOI-256 (P. S. Muirhead 2012, private communication), and KOI-1459 based on their transit profiles. The final distribution of $\mathrm{M}$ dwarf planet candidates is shown as gray circles in Figure 6. These remaining candidates are expected to have a high fidelity, allowing us to treat them as a sound, statistical ensemble.

The distribution of $\mathrm{M}$ dwarf planet candidates in Figure 6 contains 100 planets in 66 systems. There are 48 single planet 


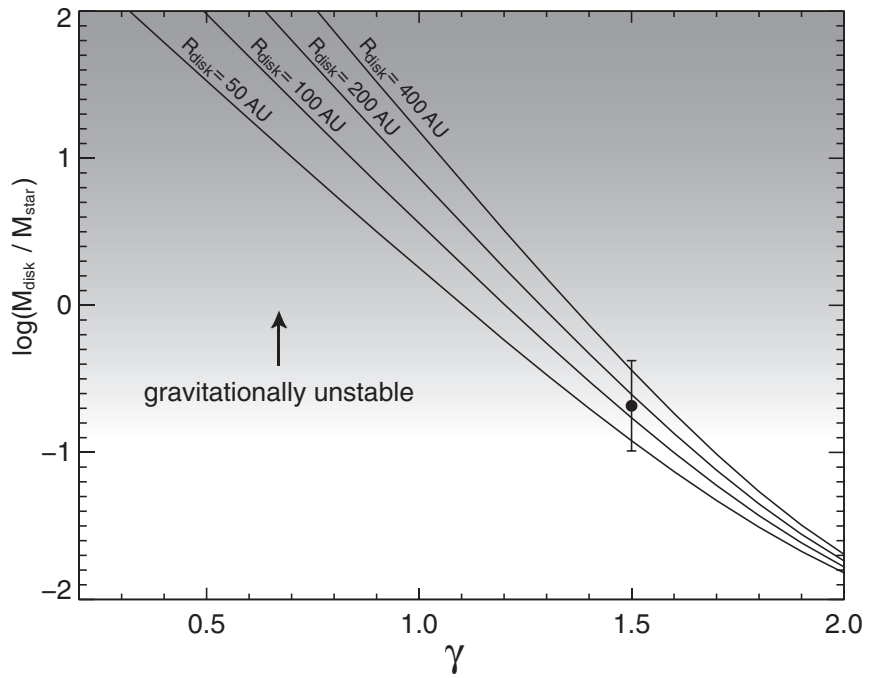

Figure 8. Inferred disk mass in units of the stellar mass for all of the $\mathrm{M}$ dwarf multi-transit systems in the Kepler sample as a function of assumed surface density profile index $\gamma$, and disk radius, $R_{\text {disk }}$. The data point and error bars show the mean and standard deviation of one such calculation for $\gamma=1.5$ and $R_{\text {disk }}=150$ AU to give a sense of the distribution of disk masses derived in each calculation. Disks with masses $\gtrsim 0.2 M_{\star}$ are expected to be gravitationally unstable.

systems, 7 double planet systems, 7 three planet systems, 3 four planet systems, and 1 five planet system-Kepler-32. Therefore, 18 systems, or $27 \%$ of the $\mathrm{M}$ dwarf KOIs, are multi-transit systems. The giant, KOI-254, is a single candidate system that constitutes $1 \%$ of all planets in the Kepler $\mathrm{M}$ dwarf sample and about $2 \%$ of all planet hosting $\mathrm{M}$ dwarfs. This planet is anomalous and we do not consider it as part of the ensemble hereafter.

The main distribution of $\mathrm{M}$ dwarf planets appears to follow a trend of increasing mass (radius) as a function of semimajor axis. Without accounting for biases, we derive the relationship $M \propto a^{0.6}$. However, for a given $\mathrm{S} / \mathrm{N}$ threshold, the lower envelope of planet candidates is expected to follow

$$
R_{\lim }=\left(\mathrm{S} / \mathrm{N} \sigma_{p}\right)^{1 / 2} R_{\star}^{3 / 4} a^{1 / 4}\left(\frac{\pi}{n}\right)^{1 / 4},
$$

where $\mathrm{S} / \mathrm{N}$ is the signal-to-noise threshold, $\sigma_{p}$ is the photometric precision, $R_{\star}$ is the stellar radius, and $n$ is the number of transits measured. For our adopted mass-radius relationship, this translates to $M_{\lim } \propto a^{0.5}$. The detection limit for an observational baseline of six quarters and an $\mathrm{S} / \mathrm{N}$ threshold of about five for a typical M1V star is shown in Figure 6 as the dashed line. This suggests that the apparent trend at the lower envelope of the distribution is due to observational biases. Also shown in Figure 6 is the semimajor axis corresponding to Kepler's observing time baseline over the first six quarters of data over which the planet candidates were selected (Batalha et al. 2012).

Although we cannot perform as detailed an analysis on the Kepler $\mathrm{M}$ dwarf planet ensemble as we have for Kepler32 , we note that positions and inferred masses of the planet candidates as a whole also imply very large disk masses within $0.1 \mathrm{AU}$ (also see Chiang \& Laughlin 2012). Figure 8 shows the reconstructed mean protoplanetary disk masses for the 18 multi-transit $\mathrm{M}$ dwarf KOIs as a function of $\gamma$. The $1 \sigma$ spread in the derived masses for any given value of $\gamma$ is approximately 0.3 dex (shown as data point and error bar). Even for $\gamma=3 / 2$, an appreciable fraction of the disks would be gravitationally
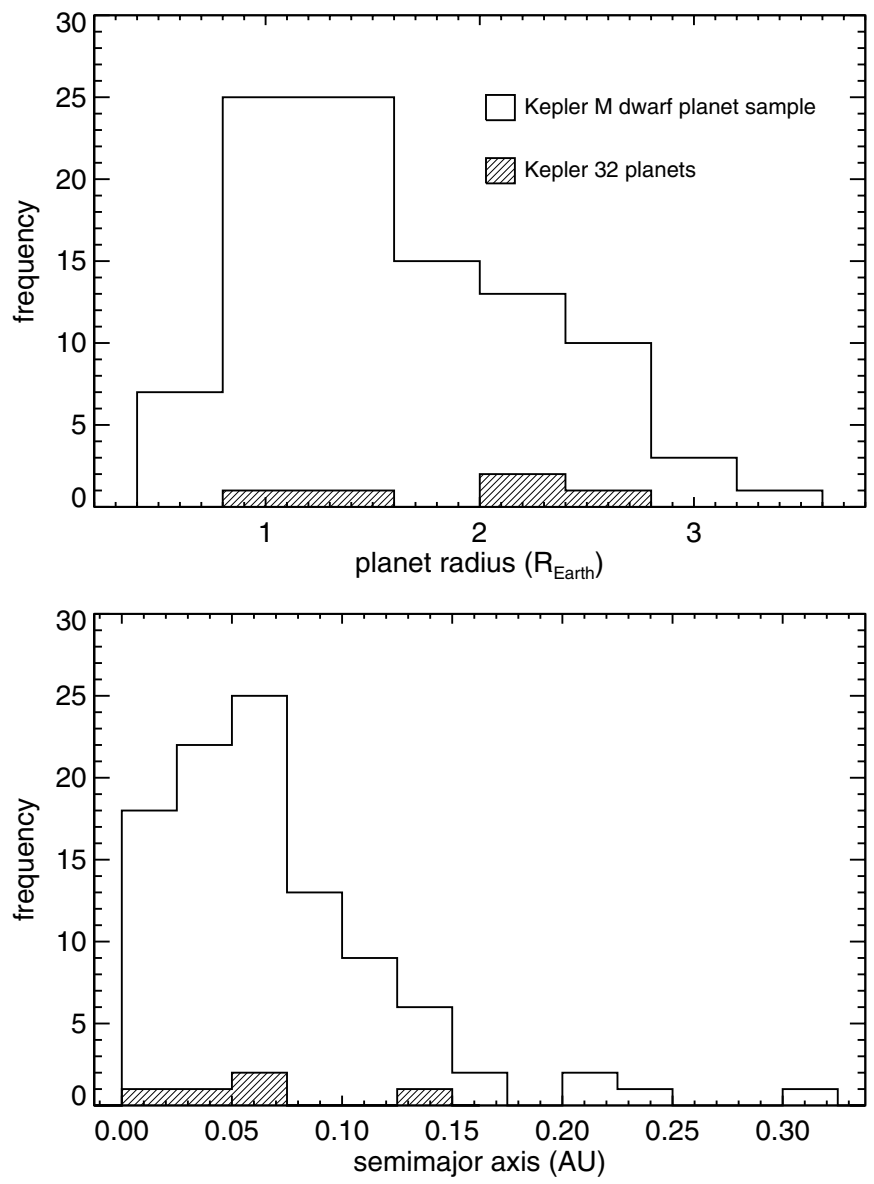

Figure 9. Distributions of Kepler $\mathrm{M}$ dwarf planet candidates as a function of planet radius and semimajor axis (blank histogram; not including KOI-254, $256,531,1152,1459$, or 1902) in comparison to the Kepler-32 planets (hashed histogram).

unstable and therefore unlikely to produce the observed planets where we see them.

We also analyze the present-day locations of the Kepler $\mathrm{M}$ dwarf planets in terms of the sublimation radius of their host star at $10 \mathrm{Myr}$ of pre-main-sequence evolution. We find that between $5 \%$ and $14 \%$ of the total number of planet candidates fall within the dust sublimation radius of their host stars for large iron grains and $1 \mu \mathrm{m}$ silicate grains, respectively, and could not have formed in place.

\subsection{Kepler-32 as a Representative of the Kepler M Dwarfs}

The Kepler-32 planets span the main distribution of the Kepler $M$ dwarf planets seen in Figure 6 and we plot in Figure 9 the locations of these planets in relation to the full distribution as a function of planet radius and semimajor axis. In both parameter spaces, the Kepler-32 planets fall well within the main distribution of planet candidates.

To further explore how representative the Kepler-32 planetary system is of the full sample of Kepler $\mathrm{M}$ dwarf planet candidates, we create an ensemble of planetary systems with the Kepler-32 system specifications oriented randomly on the sky with mutual inclinations drawn randomly from a Rayleigh distribution (Lissauer et al. 2011). We find the transit multiplicity fractions are best reproduced with a spread in mutual inclinations of $1.2 \pm 0.2$, consistent with values for the entire Kepler planet candidate ensemble (1.0-2.3; Fabrycky et al. 2012b). The discrepancies between the real and simulated distributions 

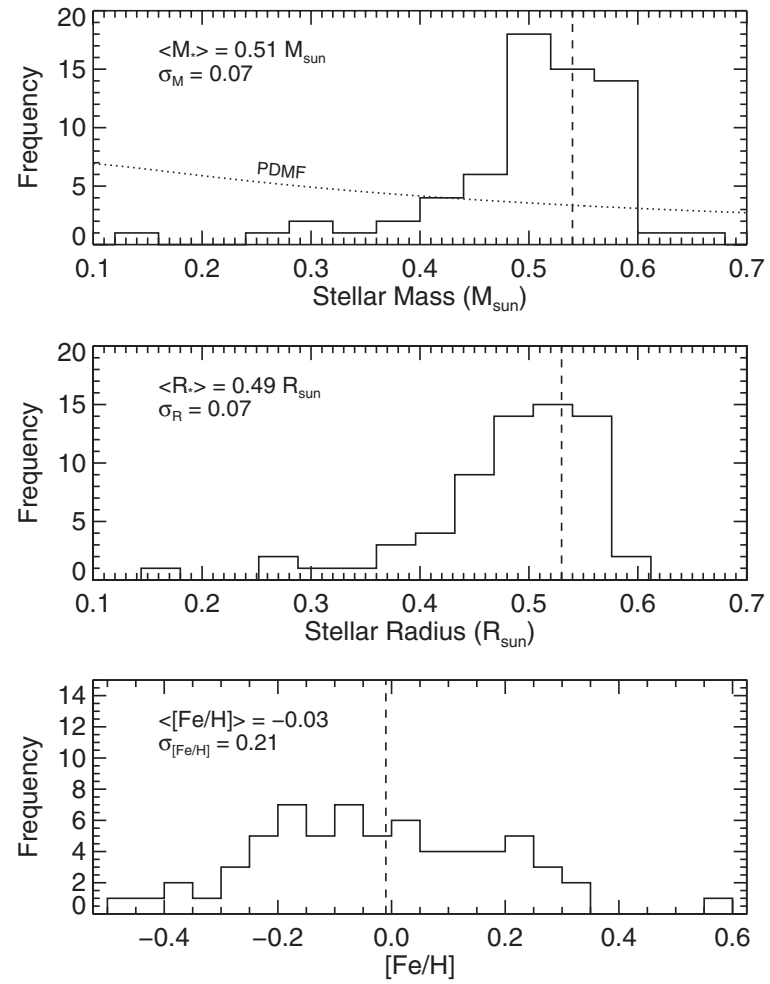

Figure 10. Distribution of stellar masses and radii for our sample of $\mathrm{M}$ dwarf planet host stars are sharply peaked around $0.53 M_{\odot}$ and $0.51 R_{\odot}$, respectively. The present-day mass function of single field stars in the Galaxy (Chabrier 2003) normalized to the number of stars in our sample is shown as the dotted line in the top panel for reference. Metallicities for our sample fall mostly between -0.4 and 0.4 . Kepler- 32 values denoted by the vertical dashed line all fall near the center of these distributions.

of transiting systems are largest for the single transit systems (55\% simulated versus $73 \%$ real). This would be expected for a situation where the typical planetary system contains less than five planets or is less compact. However, we note that the observed transit multiplicity of Kepler M dwarf systems can be recreated remarkably well assuming the case that all planetary systems are exact clones of Kepler-32.

The distributions of stellar masses, radii, and metallicities for the Kepler M dwarf planet host stars are shown in Figure 10. A narrow range of stellar mass and radius is seen peaked around 0.5 in solar units due to the magnitude limit of the sample and is shown in contrast to the present-day mass function of single stars (PDMF; Chabrier 2003) in the top panel. Kepler-32 is seen to be representative of the full sample in all quantities.

\subsection{Planet Occurrence}

The planet occurrence rate for $\mathrm{M}$ dwarf planets has been calculated to be about 0.3 for planets with radii $>2 R_{\oplus}$ and periods less than 50 days (Mann et al. 2012). However, only 28 planets of the 100 total in our Kepler M dwarf ensemble satisfy these criteria. Therefore, the total planet occurrence rate for short-period planets around $\mathrm{M}$ dwarfs is much higher than this number. The detection of planet signals within the Kepler $\mathrm{M}$ dwarf sample is not uniform, causing a systematic uncertainty in planet occurrence estimations. Since we have no way to correct for this currently, we ignore this effect, which will result in a lower limit of the occurrence rate.
The number of planets per star in our sample is estimated as

$$
f=\sum_{i}^{n_{p}} \frac{1}{\sum_{j}^{n_{\star}, i} p_{i, j}},
$$

where $j$ is the index of the sum over all stars around which planet $i$ could be detected, $n_{\star, i}$ (see, e.g., Howard et al. 2012), and

$$
p_{i, j}=\frac{R_{\star, j}}{a_{i}}
$$

is the geometric probability of detecting planet $i$ around star $j$ if eccentricity is negligible and $R_{p} / R_{\star} \ll 1$. Since the KIC stellar radii values for stars with $T_{\text {eff }} \lesssim 4500 \mathrm{~K}$ are unreliable, we randomly sample the distribution of stellar radii in Figure 10 estimated from near-infrared spectra for this calculation. Evaluating Equation (8) over our sample yields $f=1.0 \pm 0.1$ planets per star, where we use a binomial error estimate.

Though we do not perform statistical validations for all of the Kepler M dwarf planets, our sample is expected to have a higher fidelity than the total ensemble of Kepler transit signals since we vetted our sample by hand. The planet occurrence derived from our sample assuming $90 \%$ fidelity, $f_{90}$, is calculated by evaluating Equation (8) repeatedly for 90 randomly drawn planets of the 100 total. We find $f_{90}=0.9 \pm 0.1$, where the stated error is the standard deviation of the distribution of 1000 realizations. As a further check, we limit our sample to the 28 planets with $R_{p}>2 R_{\oplus}$ and $P<50$ days to obtain $f=0.26 \pm 0.05$ at the lower end of the estimations of Mann et al. (2012). This may be further evidence that our estimations are conservative.

Counting only one planet per system in Equation (8) gives the occurrence of stars with at least one planet, $0.51 \pm 0.07$ (not including KOI-254), which together with our estimate of the total occurrence gives the average number of planets per system as $\sim 2$. Stars with masses characteristic of the Kepler M dwarf sample are a factor of $\sim 1.8$ times more common than stars of $1 M_{\odot}$ (Chabrier 2003), and the planets of the Kepler32 system are representative of the planets that form around these stars. Therefore, the insights gleaned from the Kepler-32 system both show us where to look for additional planets in the solar neighborhood, and provide a template for understanding the formation of the ubiquitous compact planetary systems throughout the Galaxy.

\subsection{Summary and Future Directions}

We present a detailed analysis of the Kepler-32 planetary system which offers the rare circumstance of five transit signals. While two of the planets have previously been validated through evidence of their mutual gravitational interactions, we validate the remaining three transit signals probabilistically using observations from the W. M. Keck Observatory as constraints. This validation makes Kepler-32 the richest system of transiting planets known around an M dwarf.

Kepler-32 has a markedly compact architecture. All five planets orbit within one-third of Mercury's distance from the Sun, with the closest planet orbiting only 4.3 stellar radii from the Kepler-32 photosphere. The three middle planets lie close to a 1:2:3 period commensurability that is unlikely to be the result of chance.

Our refined stellar parameters improve the derived planetary characteristics, and aid in reconstructing this system's formation 
history. Several pieces of evidence from our analyses indicate that the Kepler-32 planets did not form where we see them today:

1. the dust sublimation radius of Kepler-32 lying outside the present-day semimajor axis of Kepler-32 f for longer than a typical protostellar disk lifetime;

2. the extremely high surface densities inferred by assuming in situ formation;

3. the limited range of gravitational influence for planetary embryos located so close to their host star;

4. the unlikely arrangement of three planets in the system near a 1:2:3 period commensurability;

5. the high volatile content of Kepler-32 b and c.

This conclusion necessitates planet migration through a disk, and our order of magnitude calculations for the migration rates of the Kepler-32 planets embedded in a typical protostellar disk suggest the presence of gas. If true, then this would limit the formation time of the Kepler-32 planets to $\lesssim 10 \mathrm{Myr}$ - the known timescales over which gaseous disks survive.

Kepler-32 is found to be representative of the full sample of 66 Kepler M dwarf host stars, and the Kepler-32 planets span the mid-line of the distribution of 100 Kepler $\mathrm{M}$ dwarf planet candidates in radius-semimajor axis space. Although we are unable to treat each system in this ensemble with the same care as for Kepler-32, we show that similar analyses applied to the ensemble give consistent results to those derived for Kepler-32. Thus, the formation scenario deduced from Kepler-32 offers a plausible blueprint for the formation of the full sample of Kepler $\mathrm{M}$ dwarf planets.

We select out $4682 \mathrm{M}$ dwarfs from the KIC that have been observed with Kepler and use their observational parameters to derive the planet occurrence rate of Kepler $\mathrm{M}$ dwarf planet candidates. We confirm that within the completeness limits of the first six quarters of Kepler data, the $\mathrm{M}$ dwarf planet candidates have an occurrence rate about three times that of solar-type stars, while the occurrence rate of all candidates around $\mathrm{M}$ dwarfs is $1.0 \pm 0.1$. We expect the fidelity of our vetted sample to be above $90 \%$. Thus, the compact systems of planets around the Kepler $\mathrm{M}$ dwarf sample are a major population of planets throughout the Galaxy amplifying the significance of the insights gleaned from Kepler-32.

At the time of this writing, there are only 37 planets confirmed to exist around $24 \mathrm{M}$ dwarfs in the Galaxy (www.exoplanets.org; Wright et al. 2011). The Kepler space telescope has revealed 100 planet candidates around the $66 \mathrm{M}$ dwarfs from which we draw our statistics. It would be of great benefit to the study of $\mathrm{M}$ dwarf planet formation to explore and validate a larger statistical sample of $\mathbf{M}$ dwarfs such that comparisons can be drawn against the detailed analyses of solar-type stars (e.g., Youdin 2011). The continued monitoring of the current sample is also important, as this will reveal trends in planet occurrence as a function of orbital period for the smallest planets.

Mass measurements of the growing numbers of confirmed M dwarf planets will also play an important role in interpreting their origins. This can be achieved with nearby $\mathrm{M}$ dwarfs using precision radial velocity measurements, or by using alternative techniques such as the amplitude of transit timing variations.

Lastly, direct imaging of the inner few AU of nearby protostellar disks will be possible in multiple wavebands with the Atacama Large Telescope Array. The modeling of this emission may be the most direct way to constrain where in the protoplanetary disk compact planetary systems form.
This work has benefited from the feedback and suggestions of many people, including but not limited to Andrew Youdin, Leslie Rogers, Peter Goldreich, Hilke Schlichting, and Nairn Baliber. We also thank the anonymous referee for a thorough review. D.C.F. acknowledges support for this work was provided by NASA through Hubble Fellowship grant HF-51272.01-A, awarded by the Space Telescope Science Institute, which is operated by the Association of Universities for Research in Astronomy, Inc., for NASA, under contract NAS 5-26555.

Some of the data presented herein were obtained at the W. M. Keck Observatory, which is operated as a scientific partnership among the California Institute of Technology, the University of California and the National Aeronautics and Space Administration. The Observatory was made possible by the generous financial support of the W. M. Keck Foundation.

This paper includes data collected by the Kepler mission. Funding for the Kepler mission is provided by the NASA Science Mission directorate.

The Robo-AO system is supported by collaborating partner institutions, the California Institute of Technology and the InterUniversity Centre for Astronomy and Astrophysics, by the National Science Foundation under grant Nos. AST-0906060 and AST-0960343, by a grant from the Mt. Cuba Astronomical Foundation and by a gift from Samuel Oschin.

\section{REFERENCES}

Agüeros, M. A., Covey, K. R., Lemonias, J. J., et al. 2011, ApJ, 740, 110 Andrews, S. M., \& Williams, J. P. 2007, ApJ, 659, 705

Andrews, S. M., Wilner, D. J., Hughes, A. M., Qi, C., \& Dullemond, C. P. 2009, ApJ, 700, 1502

Baraffe, I., Chabrier, G., Allard, F., \& Hauschildt, P. H. 1998, A\&A, 337, 403

Baranec, C., Riddle, R., Ramaprakash, A. N., et al. 2012, Proc. SPIE, 8447, 844704

Barnes, S. A. 2007, ApJ, 669, 1167

Barnes, S. A. 2010, ApJ, 722, 222

Batalha, N. M., Rowe, J. F., Bryson, F. T., et al. 2012, arXiv:1202.5852

Batygin, K., \& Morbidelli, A. 2013, AJ, 145, 1

Bochanski, J. J., Hawley, S. L., Covey, K. R., et al. 2010, AJ, 139, 2679

Borucki, W. J., Koch, D. G., Basri, G., et al. 2011a, ApJ, 728, 117

Borucki, W. J., Koch, D. G., Basri, G., et al. 2011b, ApJ, 736, 19

Bromley, B. C., \& Kenyon, S. J. 2011, ApJ, 735, 29

Brown, T. M., Latham, D. W., Everett, M. E., \& Esquerdo, G. A. 2011, AJ, 142,112

Cassan, A., Kubas, D., Beaulieu, J.-P., et al. 2012, Natur, 481, 167

Chabrier, G. 2003, PASP, 115, 763

Chambers, J. E. 1999, MNRAS, 304, 793

Chambers, J. E., \& Wetherill, G. W. 1998, Icar, 136, 304

Chambers, J. E., Wetherill, G. W., \& Boss, A. P. 1996, Icar, 119, 261

Chiang, E., \& Laughlin, G. 2012, arXiv:1211.1673

Cresswell, P., \& Nelson, R. P. 2008, A\&A, 482, 677

Crida, A., Morbidelli, A., \& Masset, F. 2006, Icar, 181, 587

Delfosse, X., Forveille, T., Ségransan, D., et al. 2000, A\&A, 364, 217

Eisner, J. A. 2012, ApJ, 755, 23

Fabrycky, D. C., Ford, E. B., Steffen, J. H., et al. 2012a, ApJ, 750, 114

Fabrycky, D. C., Lissauer, J. J., Ragozzine, D., et al. 2012b, arXiv:1202.6328

Fortney, J. J., Marley, M. S., \& Barnes, J. W. 2007, ApJ, 659, 1661

Gladman, B. 1993, Icar, 106, 247

Goldreich, P., \& Tremaine, S. 1979, ApJ, 233, 857

Gould, A., Pepper, J., \& DePoy, D. L. 2003, ApJ, 594, 533

Hansen, B. M. S., \& Murray, N. 2012, ApJ, 751, 158

Hayashi, C. 1981, PThPS, 70, 35

Hodgkin, S. T., \& Pye, J. P. 1994, MNRAS, 267, 840

Howard, A. W., Marcy, G. W., Bryson, S. T., et al. 2012, ApJS, 201, 15

Isella, A., Carpenter, J. M., \& Sargent, A. I. 2009, ApJ, 701, 260

Isella, A., Testi, L., \& Natta, A. 2006, A\&A, 451, 951

Jenkins, J. M., Caldwell, D. A., Chandrasekaran, H., et al. 2010a, ApJL, 713, L87

Jenkins, J. M., Chandrasekaran, H., McCauliff, S. D., et al. 2010b, Proc. SPIE, 7740,10

Jester, S., Schneider, D. P., Richards, G. T., et al. 2005, AJ, 130, 873 
Johnson, J. A., Aller, K. M., Howard, A. W., \& Crepp, J. R. 2010a, PASP, 122,905

Johnson, J. A., Gazak, J. Z., Apps, K., et al. 2012, AJ, 143, 111

Johnson, J. A., Howard, A. W., Marcy, G. W., et al. 2010b, PASP, 122, 149

Kasting, J. F., Whitmire, D. P., \& Reynolds, R. T. 1993, Icar, 101, 108

Kennedy, G. M., \& Kenyon, S. J. 2008, ApJ, 673, 502

Kirsh, D. R., Duncan, M., Brasser, R., \& Levison, H. F. 2009, Icar, 199, 197

Kley, W., \& Nelson, R. P. 2012, ARA\&A, 50, 211

Koch, D. G., Borucki, W. J., Basri, G., et al. 2010, ApJL, 713, L79

Kokubo, E., \& Ida, S. 1998, Icar, 131, 171

Laughlin, G., Bodenheimer, P., \& Adams, F. C. 2004, ApJL, 612, L73

Lecavelier Des Etangs, A. 2007, A\&A, 461, 1185

Levison, H. F., Morbidelli, A., Gomes, R., \& Backman, D. 2007, in Protostars and Planets V, ed. B. Reipurth, D. Jewitt, \& K. Keil (Tucson, AZ: Univ. Arizona Press), 669

Lin, D. N. C., \& Papaloizou, J. 1979, MNRAS, 186, 799

Lissauer, J. J. 1987, Icar, 69, 249

Lissauer, J. J. 2007, ApJL, 660, L149

Lissauer, J. J., Marcy, G. W., Rowe, J. F., et al. 2012, ApJ, 750, 112

Lissauer, J. J., Ragozzine, D., Fabrycky, D. C., et al. 2011, ApJS, 197, 8

Lithwick, Y., \& Wu, Y. 2012, ApJL, 756, L11

Lithwick, Y., Xie, J., \& Wu, Y. 2012, ApJL, 761, L122

Mann, A. W., Gaidos, E., Lépine, S., \& Hilton, E. J. 2012, ApJ, 753, 90

Masset, F. S., D’Angelo, G., \& Kley, W. 2006, ApJ, 652, 730
Moore, A., Hasan, I., \& Quillen, A. 2012, arXiv:1207.6601

Morton, T. D. 2012, ApJ, 761, 6

Morton, T. D., \& Johnson, J. A. 2011, ApJ, 738, 170

Muirhead, P. S., Hamren, K., Schlawin, E., et al. 2012a, ApJL, 750, L37

Muirhead, P. S., Johnson, J. A., Apps, K., et al. 2012b, ApJ, 747, 144

Murray, C. D., \& Dermott, S. F. 1999, Solar System Dynamics (Cambridge: Cambridge University Press)

Nidever, D. L., Marcy, G. W., Butler, R. P., Fischer, D. A., \& Vogt, S. S. 2002, ApJS, 141, 503

Nutzman, P., \& Charbonneau, D. 2008, PASP, 120, 317

Pollack, J. B., Hollenbach, D., Beckwith, S., et al. 1994, ApJ, 421, 615

Rogers, L. A., Bodenheimer, P., Lissauer, J. J., \& Seager, S. 2011, ApJ, 738,59

Smith, A. W., \& Lissauer, J. J. 2009, Icar, 201, 381

Smith, R. J., Clark, P. C., \& Bonnell, I. A. 2009, MNRAS, 396, 830

Tarter, J. C., Backus, P. R., Mancinelli, R., et al. 2007, AsBio, 7, 30

Vogt, S. S., Allen, S. L., Bigelow, B. C., et al. 1994, Proc. SPIE, 2198, 362

Weidenschilling, S. J. 1977, Ap\&SS, 51, 153

West, A. A., Walkowicz, L. M., \& Hawley, S. L. 2005, PASP, 117, 706

Wetherill, G. W. 1990, AREPS, 18, 205

Williams, J. P., \& Cieza, L. A. 2011, ARA\&A, 49, 67

Wright, J. T., Fakhouri, O., Marcy, G. W., et al. 2011, PASP, 123, 412

Wu, Y., \& Lithwick, Y. 2012, arXiv:1210.7810

Youdin, A. N. 2011, ApJ, 742, 38 